Commun. Fac. Sci. Univ. Ank. Ser. A1 Math. Stat.

Volume 68, Number 2, Pages 22642271(2019)

DOI: $10.31801 /$ cfsuasmas. 562214

ISSN 1303-5991 E-ISSN 2618-6470

http://communications.science.ankara.edu.tr/index.php?series=A1

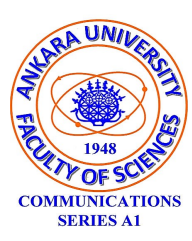

\title{
MEAN ERGODIC TYPE THEOREMS
}

\author{
G. OĞUZ AND C. ORHAN
}

\begin{abstract}
Let $T$ be a bounded linear operator on a Banach space $X$. Replacing the Cesàro matrix by a regular matrix $A=\left(a_{n j}\right)$ Cohen studied a mean ergodic theorem. In the present paper we extend his result by taking a sequence of infinite matrices $\mathcal{A}=\left(A^{(i)}\right)$ that contains both convergence and almost convergence. This result also yields an $\mathcal{A}$-ergodic decomposition. When $T$ is power bounded we give a characterization for $T$ to be $\mathcal{A}$-ergodic.
\end{abstract}

\section{INTRODUCTION}

Let $X$ be a Banach space and $T$ be a bounded linear operator on $X$ into itself. By $M_{n}(T)$ we denote the Cesàro averages of $T$ given by $M_{n}(T):=\frac{1}{n+1} \sum_{j=0}^{n} T^{j}$. An operator $T \in B(X)$ is called mean ergodic, respectively uniformly ergodic, if $\left\{M_{n}(T)\right\}$ is strongly, respectively uniformly, convergent in $B(X)$. Cohen [3] considered the problem of determining a class of regular matrices $A=\left(a_{n j}\right)$ for which

$$
L_{n}:=\sum_{j=1}^{\infty} a_{n j} T^{j}
$$

converges strongly to an element invariant under $T$. It is the case when $\left\{L_{n} x: n \in\right.$ $\mathbb{N}\}$ is weakly compact and $\lim _{k} \sum_{j=k}^{\infty}\left|a_{n, j+1}-a_{n j}\right|=0$ uniformly in $n$ (see also [11]).

Observe that Cohen's result is an extension of the mean ergodic theorems due to von Nuemann [10, F. Riesz [8] and K. Yosida [12].

In the present paper, replacing the matrix $A=\left(a_{n j}\right)$ by a sequence of infinite matrices $\left(A^{(i)}\right)=\left(a_{n j}^{(i)}\right)$ we study results in an analogy of Cohen.

Now, we give some basic notations concerning the sequence of infinite matrices. Let $\mathcal{A}$ be a sequence of infinite matrices $\left(A^{(i)}\right)=\left(a_{n j}^{(i)}\right)$. Given a sequence $x=\left(x_{j}\right)$

Received by the editors: May 09, 2019; Accepted: June 17, 2019.

2010 Mathematics Subject Classification. 47A35, 46B15, $40 \mathrm{C} 05$.

Key words and phrases. Infinite matrices, almost convergence, ergodic theorems. 
we write

$$
A_{n}^{(i)} x=\sum_{j=1}^{\infty} a_{n j}^{(i)} x_{j}
$$

if it exists for each $n$ and $i \geq 0$. The sequence $\left(x_{j}\right)$ is said to be summable to the value $s$ by the method $\mathcal{A}$ if

$$
A_{n}^{(i)} x \rightarrow s \quad(n \rightarrow \infty, \text { uniformly in } i) .
$$

If (1) holds, we write $x \rightarrow s(\mathcal{A})$.

The method $\mathcal{A}$ is called conservative if $x \rightarrow s$ implies $x \rightarrow s^{\prime}(\mathcal{A})$. If $\mathcal{A}$ is conservative and $s=s^{\prime}$, we say that $\mathcal{A}$ is regular. We now recall a theorem which characterizes the regularity of the sequences of infinite matrices.

Theorem 1 ([2, 9]). Let $\mathcal{A}$ be the sequence of infinite matrices $\left(A^{(i)}\right)=\left(a_{n j}^{(i)}\right)$. Then, $\mathcal{A}$ is regular if and only if the following conditions hold:

(1) $\sum_{j}\left|a_{n j}^{(i)}\right|<\infty, \quad($ for all $n$, for all $i)$,

(2) There exists an integer $m$ such that $\sup _{i \geq 0, n \geq m} \sum_{j}\left|a_{n j}^{(i)}\right|<\infty$,

(3) for all $j, \lim a_{n j}^{(i)}=0$, (uniformly in $i$ ),

(4) $\lim _{n} \sum_{j} a_{n j}^{(i)}=1$, (uniformly in $i$ ).

In addition, we write

$$
\|\mathcal{A}\|:=\sup _{n, i} \sum_{j}\left|a_{n j}^{(i)}\right|
$$

and $\|\mathcal{A}\|<\infty$ to mean that, there exists a constant $M$ such that $\sum_{j}\left|a_{n j}^{(i)}\right| \leq M$, (for all $n$, for all $i$ ) and the series $\sum_{j} a_{n j}^{(i)}$ converges uniformly in $i$ for each $n$.

Throughout the paper we assume that the sequence of matrices $\left(A^{(i)}\right)=\left(a_{n j}^{(i)}\right)$ satisfies the following conditions:

(i) $\mathcal{A}$ is regular,

(ii) $\|\mathcal{A}\|<\infty$,

(iii) $\limsup _{k} \sum_{i, n}^{\infty}\left|a_{n, j+1}^{(i)}-a_{n j}^{(i)}\right|=0$.

\section{MAIN RESULTS}

In this section, using a sequence of infinite matrices we give a theorem analogous to one of Cohen [3].

We now present a lemma which will be used in the proof of the main theorem. 
Lemma 2. Let $T$ and $A_{n}^{(i)}$ be bounded linear operators on a Banach space $X$ into itself such that $T A_{n}^{(i)}=A_{n}^{(i)} T$ for all $n$ and $i$. If

$$
\left.\lim _{n \rightarrow \infty} A_{n}^{(i)}(x-T x)=0, \quad \text { (uniformly in } i\right),
$$

and

$$
A_{n}^{(i)} x \rightarrow x_{0}(w), \quad(n \rightarrow \infty, \text { uniformly in } i),
$$

then $T x_{0}=x_{0}$, where $(w)$ indicates the weak convergence.

Proof. By $X^{\prime}$ we denote the dual space of $X$. Let $f \in X^{\prime}$. Then, by weak convergence (uniformly in $i)$ of $\left(A_{n}^{(i)} x\right)$ we have

$$
\limsup _{n} f\left(A_{n}^{(i)} x-x_{0}\right)=0 .
$$

Since $T$ is a linear and continuous operator on $X$, we also have

$$
\lim _{n} \sup _{i} f\left(T A_{n}^{(i)} x-T x_{0}\right)=0 .
$$

It follows from (3) and the fact that $f \in X^{\prime}$,

$$
\lim _{n \rightarrow \infty} \sup _{i} f\left(A_{n}^{(i)} x-A_{n}^{(i)} T x\right)=0 .
$$

Using the commutativity $T A_{n}^{(i)}=A_{n}^{(i)} T$ for each $n$ and $i$, one may write

$$
f\left(x_{0}-T x_{0}\right)=f\left(x_{0}-A_{n}^{(i)} x\right)+f\left(A_{n}^{(i)} x-A_{n}^{(i)} T x\right)+f\left(T A_{n}^{(i)} x-T x_{0}\right) .
$$

Applying the operator $\lim _{n} \sup _{i}$ to both sides of (7) we get that

$$
\begin{aligned}
\left|\limsup _{i} f\left(x_{0}-T x_{0}\right)\right| \leq & \left|\limsup _{n} \sup _{i} f\left(x_{0}-A_{n}^{(i)} x\right)\right|+\left|\limsup _{n} f\left(A_{n}^{(i)} x-A_{n}^{(i)} T x\right)\right| \\
& +\left|\limsup _{n} \sup _{i} f\left(T A_{n}^{(i)} x-T x_{0}\right)\right| .
\end{aligned}
$$

Then by (4), (5), (6) and (8), we conclude that $f\left(x_{0}-T x_{0}\right)=0$ for all $f \in X^{\prime}$. This implies that $T x_{0}=x_{0}$.

We now present the main result of the paper.

Theorem 3. Let $X$ be a Banach space and $T: X \rightarrow X$ be a bounded linear operator. Suppose that there exists an $H>0$ such that $\left\|T^{j}\right\| \leq H$ for all $j \in \mathbb{N}$. Suppose that the sequence of infinite matrices $\left(A^{(i)}\right)=\left(a_{n j}^{(i)}\right)$ satisfies the conditions (i)-(iii) and define $A_{n}^{(i)} x=\sum_{j=1}^{\infty} a_{n j}^{(i)} T^{j} x$. Assume that there exists a subsequence $\left\{A_{n_{p}}^{(i)} x\right\} \subset\left\{A_{n}^{(i)} x\right\}$ such that

$$
\limsup _{p} A_{i}^{(i)} x=x_{0}(w),
$$


where $x_{0} \in X$. Then, $T x_{0}=x_{0}$ and $\lim _{n \rightarrow \infty} A_{n}^{(i)} x=x_{0}$ (uniformly in $i$ ). Denote by $P$ the strong limit in $B(X)$ of $\left\{A_{n}^{(i)} x\right\}$. Then it is the projection onto the space $N(I-T)$ of $T$-fixed points corresponding to the ergodic decomposition $X=\overline{R(I-T)} \oplus N(I-T)$ and $P=P^{2}=T P=P T$.

Proof. From the hypothesis there exists an $H>0$ such that $\left\|T^{j}\right\| \leq H$ for all $j \in \mathbb{N}$. Since $\|\mathcal{A}\|<\infty$, for $x \in X$ we have

$$
\begin{aligned}
\left\|A_{n}^{(i)} x\right\|=\left\|\sum_{j=1}^{\infty} a_{n j}^{(i)} T^{j} x\right\| & \leq H\|x\| \sum_{j=1}^{\infty}\left|a_{n j}^{(i)}\right| \\
& \leq H\|x\| \sup _{n, i} \sum_{j=1}^{\infty}\left|a_{n j}^{(i)}\right|<H\|x\|\|\mathcal{A}\| .
\end{aligned}
$$

Since $X$ is complete, each $\left\{A_{n}^{(i)} x\right\}$ is defined on $X$. By taking supremum over $\|x\|=1$ in both sides of (10), we get, for all $n$ and $i$, that

$$
\left\|A_{n}^{(i)}\right\| \leq H\|\mathcal{A}\| \text {. }
$$

Also we have

$$
T A_{n}^{(i)} x=\sum_{j=1}^{\infty} a_{n j}^{(i)} T^{j+1} x=A_{n}^{(i)} T x .
$$

By the hypothesis, we have for any $\varepsilon>0$ that there exists a $k_{0}=k_{0}(\varepsilon) \in \mathbb{N}$ such that for all $k \geq k_{0}$

$$
\sup _{i, n} \sum_{j=k}^{\infty}\left|a_{n, j+1}^{(i)}-a_{n j}^{(i)}\right|<\varepsilon .
$$

Hence, we get, for each $x \in X$, that

$$
\begin{aligned}
\left\|A_{n}^{(i)}(x-T x)\right\| & =\left\|a_{n 1}^{(i)} T x+\sum_{j=1}^{\infty}\left(a_{n, j+1}^{(i)}-a_{n j}^{(i)}\right) T^{j+1} x\right\| \\
& \left.\leq H\|x\| \sup _{i}\left|a_{n 1}^{(i)}\right|+\sup _{i} \sum_{j=1}^{k_{0}-1}\left|a_{n, j+1}^{(i)}-a_{n j}^{(i)}\right|+\sup _{i, n} \sum_{j=k_{0}}^{\infty}\left|a_{n, j+1}^{(i)}-a_{n j}^{(i)}\right|\right) \\
& \leq H\|x\|\left(2 \sup _{i} \sum_{j=1}^{k_{0}}\left|a_{n j}^{(i)}\right|+\varepsilon\right)
\end{aligned}
$$

Then, for $n>n_{\varepsilon}$ we also have $\sup _{i} \sum_{j=1}^{k_{0}}\left|a_{n j}^{(i)}\right|<\varepsilon$ which yields

$$
\left\|A_{n}^{(i)}(x-T x)\right\| \leq H\|x\| 3 \varepsilon
$$


This implies

$$
\left.\lim _{n \rightarrow \infty} A_{n}^{(i)}(x-T x)=0, \quad \text { (uniformly in } i\right) .
$$

Furthermore, from (9), 12 and $(13)$, the conditions of Lemma 2 are satisfied. Thus, one can get $T x_{0}=x_{0}$.

Now, we consider the linear subspace $X_{0}$ spanned by $x-T x$ for $x \in X$. We will show that $x_{0}-x \in X_{0}$. To achieve this, we follow the idea given by Cohen 3 . Assume that $x_{0}-x \notin X_{0}$. Then, one can easily see that there exists an $f \in X^{\prime}$ such that

$$
f(u)=0, \quad u \in X_{0} ; \quad f\left(x-x_{0}\right)=1 .
$$

Since $T^{k} x-T^{k+1} x \in X_{0}$ for $k=0,1,2, \ldots$, we have $f\left(T^{k} x-T^{k+1} x\right)=0$. Then, it is easy to show that $f\left(x-T^{j} x\right)=0$. So we obtain

$$
f(x)=f\left(T^{j} x\right), \quad j=1,2, \ldots
$$

Moreover, from (11) and (13), it follows that

$$
\limsup _{n} A_{i}^{(i)} u=0, \quad u \in X_{0} .
$$

Since $f \in X^{\prime}$, one can get by (14) that

$$
f\left(A_{n}^{(i)} x\right)=\sum_{j=1}^{\infty} a_{n j}^{(i)} f\left(T^{j} x\right)=\left(\sum_{j=1}^{\infty} a_{n j}^{(i)}\right) f(x)
$$

which yields

$$
\limsup _{n} f\left(A_{n}^{(i)} x\right)=f(x)
$$

By $(9)$ and $(16)$ we obtain

$$
\begin{aligned}
0=\limsup _{p} \sup _{i} f\left(A_{n_{p}}^{(i)} x-x_{0}\right) & =\limsup _{p} \sup _{i}\left(f\left(A_{n_{p}}^{(i)} x\right)-f\left(x_{0}\right)\right) \\
& =f(x)-f\left(x_{0}\right)=f\left(x-x_{0}\right) .
\end{aligned}
$$

This is a contradiction. Then we necessarily have $x_{0}-x \in X_{0}$. Since $T x_{0}=x_{0}$ we have $T^{j} x_{0}=x_{0}$ for $j=1,2, \ldots$. Hence we have

$$
A_{n}^{(i)} x_{0}=\sum_{j=1}^{\infty} a_{n j}^{(i)} T^{j} x_{0}=\left(\sum_{j=1}^{\infty} a_{n j}^{(i)}\right) x_{0}
$$

from which we immediately get

$$
\lim _{n} \sup _{i} A_{n}^{(i)} x_{0}=x_{0} .
$$

Since $x=x_{0}+\left(x-x_{0}\right)$, we get from 15$)$ and 18$)$ that

$$
\limsup _{n} A_{n}^{(i)} x=x_{0}
$$

which proves the first claim.

We can write $x=x_{0}+\left(x-x_{0}\right)$ such that $x_{0} \in N(I-T)$ and $\left(x-x_{0}\right) \in R(I-T) \subset$ 
$\overline{R(I-T)}$. Now let $\varepsilon>0$ and let $z \in \overline{R(I-T)} \cap N(I-T)$. Following [4] we then have $\|z-(u-T u)\|<\varepsilon /(3 H\|\mathcal{A}\|)$ for $u \in X$. Hence

$$
\left\|A_{n}^{(i)}(z-(u-T u))\right\|<\left\|\sum_{j=1}^{\infty} a_{n j}^{(i)} T^{j}\right\|\|z-(u-T u)\|<\frac{\varepsilon}{3} .
$$

Since $z \in \overline{R(I-T)} \cap N(I-T)$, we observe that

$$
A_{n}^{(i)} z=\sum_{j=1}^{\infty} a_{n j}^{(i)} T^{j} z=\sum_{j=1}^{\infty} a_{n j}^{(i)} z
$$

from which we get

$$
\limsup _{i} A_{n}^{(i)} z=z
$$

By (15), 19) and 21), we conclude that

$\|z\|=\left\|z-A_{n}^{(i)} z+A_{n}^{(i)} z\right\| \leq\left\|z-A_{n}^{(i)} z\right\|+\left\|A_{n}^{(i)}(z-(u-T u))\right\|+\left\|A_{n}^{(i)}(u-T u)\right\|<\varepsilon$.

Hence, we find that $\overline{R(I-T)} \cap N(I-T)=\{0\}$, which implies that

$$
X=\overline{R(I-T)} \oplus N(I-T) .
$$

On the other hand, we know that $\limsup _{n} A_{n}^{(i)} x=x_{0}$. Let $P x:=\limsup _{n} A_{n}^{(i)} x=x_{0}$. Then, since $T x_{0}=x_{0}$ and $P x=x_{0}$ one can obtain, for all $x \in X$, that

$$
T x_{0}=T P x=x_{0}=P x
$$

which yields $T P=P$. Also, we have $T^{j} P=P$ for all $j \in \mathbb{N}$. Hence, we observe that

$$
A_{n}^{(i)} P x=\sum_{j=1}^{\infty} a_{n j}^{(i)} T^{j} P x=\sum_{j=1}^{\infty} a_{n j}^{(i)} P x
$$

Applying the operator $\limsup _{n} \sup _{i}$ to both sides we find $P^{2}=P$.

In addition, from 15 we obtain $P x=P T x$ for all $x \in X$, that is $P=P T$. This concludes the proof.

Remark 4. If we define the sequence of matrices $\left(A^{(i)}\right)=\left(a_{n j}^{(i)}\right)$ by

$$
a_{n j}^{(i)}=\left\{\begin{array}{cc}
\frac{1}{n+1} & , \quad i \leq j \leq i+n, \\
0 & , \quad \text { otherwise }
\end{array}\right.
$$

then $\mathcal{A}$ reduces to almost convergence method of Lorentz [6]. Observe that $\left(a_{n j}^{(i)}\right)$ defined as above satisfies the conditions (i)-(iii) imposed in Section 1. Some results concerning the almost convergence of the sequence of operators may be found in [1] and [7. 
Given a sequence $\mathcal{A}$ of matrices $\left(A^{(i)}\right)=\left(a_{n j}^{(i)}\right)$, if the limit of $\left\{A_{n}^{(i)} x\right\}$ exists then we call the operator $T$ an $\mathcal{A}$-ergodic operator. Motivated by that of Proposition 2.2 in 5 we have the following

Theorem 5. Let $X$ be a Banach space, $T$ be a bounded linear operator on $X$ into itself. Assume that there exists an $H>0$ such that $\left\|T^{j}\right\| \leq H$ for all $j \in \mathbb{N}$. Let $\left(A^{(i)}\right)=\left(a_{n j}^{(i)}\right)$ be a sequence of infinite matrices satisfying the conditions (i)-(iii). Then, the operator $T$ is $\mathcal{A}$-ergodic if and only if $(I-T) \overline{(I-T) X}=(I-T) X$.

Proof. Let the operator $T$ be $\mathcal{A}$-ergodic. Then, by Theorem 3 we have

$$
X=\overline{R(I-T)} \oplus N(I-T) .
$$

The necessity is proved by applying the operator $(I-T)$.

Assume that $(I-T) \overline{(I-T) X}=(I-T) X$. We have, for $x \in N(I-T)$, that

$$
A_{n}^{(i)} x=\sum_{j=1}^{\infty} a_{n j}^{(i)} T^{j} x=\sum_{j=1}^{\infty} a_{n j}^{(i)} x .
$$

Hence, we get

$$
\left\|A_{n}^{(i)} x-x\right\| \rightarrow 0, \quad(n \rightarrow \infty, \text { uniformly in } i) .
$$

Now, let $x \in \overline{R(I-T)}$. Hence, there exists $x_{k} \in R(I-T)$ so that $x_{k} \rightarrow x$. One can get

$$
\left\|A_{n}^{(i)} x\right\| \leq\left\|A_{n}^{(i)} x_{k}\right\|+\left\|A_{n}^{(i)}\left(x_{k}-x\right)\right\| .
$$

If we choose $k$ in order to make $\left\|x_{k}-x\right\|$ sufficiently small, we find that $\left\|A_{n}\left(x_{k}-x\right)\right\|$ is also sufficiently small (no matter what $n$ may be) because of the fact that $\mathcal{A}$ satisfies (ii) and $T$ is power bounded.Combining this with 15 , we observe, for $x \in \overline{R(I-T)}$, that

$$
\left\|A_{n}^{(i)} x\right\| \rightarrow 0, \quad(n \rightarrow \infty, \text { uniformly in } i) .
$$

Thus, by 22 and 23 the sequence $\left\{A_{n}^{(i)}\right\}$ is strongly convergent on $\overline{R(I-T)} \oplus N(I-T)$. Since $(I-T) \overline{(I-T) X}=(I-T) X$, for $y \in X$ there exists $z \in \overline{R(I-T)}$ such that $(I-T) z=(I-T) y$. We then get $h=y-z \in N(I-T)$. Since we have $y=h+z$ such that $h \in N(I-T)$ and $z \in \overline{R(I-T)}$, the proof is completed.

\section{REFERENCES}

[1] Aleman, A. and Suciu, L., On ergodic operator means in Banach spaces, Integr. Equ. Oper. Theory 85, (2016), 259-287.

[2] Bell, H.T., Order summability and almost convergence, Proc. Amer. Math. Soc., 38 (3), (1973), 548-552.

[3] Cohen, L.W., On the mean ergodic theorem, Ann. Math. (3), 41, (1940), 505-509.

[4] Krengel, U., Ergodic Theorems, de Gruyter Studies in Mathematics vol 6, Walter de Gruyter \& Co., Berlin, 1985. 
[5] Lin, M., Shoikhet, D. and Suciu L., Remaks on uniform ergodic theorems, Acta Sci. Math. (Szeged) 81, (2015), 251-283

[6] Lorentz, G. G., A contribution to the theory of divergent sequences, Acta Math. 80, (1948), 167-190.

[7] Nanda, S., Ergodic theory and almost convergence, Bull. Math, de la Soc. Sci. Math, de la R. S. de Roumanie 26, (1982), 339-343.

[8] Riesz, F., Some mean ergodic theorems, J. Lond. Math. Soc. 13, (1938), 274.

[9] Stieglitz, M., Eine verallgen meinerung des Begriffs Fastkonvergenz, Math. Japon. 18, (1973), 53-70.

[10] von Neumann, J., Proof of the quasi-ergodic hypothesis, Proc. Nat. Acad. Sci. USA, 18, (1932), 70-82.

[11] Yoshimoto, T., Ergodic theorems and summability methods, Quart. J. Math. 38 (3), (1987), 367-379.

[12] Yosida, K., Mean ergodic theorem in Banach space, Proc. Imp. Acad. Tokyo, 14, (1938), 292-294.

Current address: G. Oğuz: Ankara University, Faculty of Science, Department of Mathematics, 06100, Tandoğan, Ankara, Turkey.

E-mail address: gencayyoguzz@gmail.com

ORCID Address: https://orcid.org/0000-0003-4077-1080

Current address: C. Orhan: Ankara University, Faculty of Science, Department of Mathematics, 06100, Tandoğan, Ankara, Turkey.

E-mail address: orhan@science.ankara.edu.tr

ORCID Address: https://orcid.org/0000-0002-3558-4945 